WellBeing International

WBI Studies Repository

7-2014

\title{
Male Brush-Turkeys Attempt Sexual Coercion in Unusual Circumstances
}

\author{
David A. Wells \\ Macquarie University \\ Darryl N. Jones \\ Griffith University \\ David Bulger \\ Macquarie University \\ Culum Brown \\ Macquarie University
}

Follow this and additional works at: https://www.wellbeingintlstudiesrepository.org/repbeh

Part of the Animal Studies Commons, Behavior and Ethology Commons, and the Comparative Psychology Commons

\section{Recommended Citation}

Wells, D. A., Jones, D. N., Bulger, D., \& Brown, C. (2014). Male brush-turkeys attempt sexual coercion in unusual circumstances. Behavioural processes, 106, 180-186.

This material is brought to you for free and open access by WellBeing International. It has been accepted for inclusion by an authorized administrator of the WBI Studies Repository. For more information, please contact wbisr-info@wellbeingintl.org.

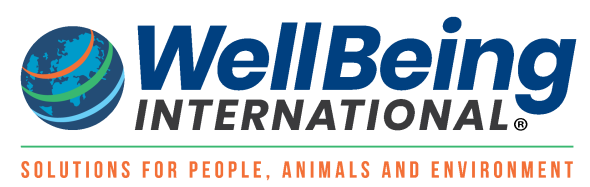




\title{
Male Brush-Turkeys Attempt Sexual Coercion in Unusual Circumstances
}

\author{
David A. Wells ${ }^{1}$, Darryl N. Jones ${ }^{2}$, David Bulger ${ }^{1}$, Culum Brown $^{1}$ \\ ${ }^{1}$ Macquarie University \\ ${ }^{2}$ Griffith University
}

\section{KEYWORDS}

Alectura lathami, behavioural syndrome, megapode, sexual coercion, sexual harassment, sexual selection

\begin{abstract}
Sexual coercion by males is generally understood to have three forms: forced copulation, harassment and intimidation. We studied Australian brush-turkeys, Alectura lathami, to determine whether some male behaviours towards females at incubation mounds could be classified as aggressive, whether males were attempting sexual coercion and, if so, whether the coercion was successful. We found that some male behaviours towards females were significantly more likely to be followed by the cessation of female mound activity, and hence could be classified as aggressive, while others were significantly more likely to be followed by the commencement of female mound activity, and hence could be classified as enticing. Copulation was preceded by higher rates of male enticement and by higher rates of certain types of male aggression. It therefore seemed that males were attempting sexual coercion. There was little evidence, however, that this combination of coercion and enticement was successful in obtaining copulations. While forced copulation did occur, it was infrequent, and no evidence could be found for intimidation. We conclude that harassment is the primary form of sexual coercion by male brush-turkeys. Although sexual coercion is understood to be a sub-optimal tactic, brush-turkey sexual coercion was employed as a primary tactic by dominant males who owned incubation mounds. One possible explanation for this apparent paradox is that aggression is the default solution for social conflicts in this species, and hence can be interpreted as a behavioural syndrome.
\end{abstract}

\section{Introduction}

In most sexual organisms, males are more eager to mate than females (Trivers, 1972). This difference typically plays out as male-male conflict over mating opportunities (intrasexual competition) or as males enhancing their prospects of being chosen as mates by females (intersexual mate choice). In many circumstances, however, it can play out as males forcing females to mate, i.e. intersexual coercion or sexual coercion (Clutton-Brock and Parker, 1995; Smuts and Smuts, 1993). The term 'sexual coercion' usually refers to coercive behaviour directed specifically at obtaining copulations and does not include other male behaviour which may also involve mating-related coercion, such as sequestering females to form a harem or mate-guarding of individual females (Clutton-Brock and Parker, 1995). Although 
behaviours such as infanticide are included in some usages (e.g. Smuts and Smuts, 1993), three nonexclusive forms of sexual coercion are generally recognized: forced copulation; harassment, i.e. males seeking to enhance their immediate mating success by repeatedly attempting to copulate; and intimidation, i.e. males attempting to enhance their future mating success by punishing females who refuse to mate with them (Clutton-Brock and Parker, 1995).

Sexual coercion may be employed by males of any dominance status, but it is typically a secondary or alternative mating tactic. For example, male marine turtles, Chelonia mydas, supplement courtship with harassment (Lee and Hays, 2004). In many species, sexual coercion is characteristically employed by subordinate males. While dominant male bighorn sheep, Ovis canadensis, for example, defend a single estrus ewe, subordinate rams attempt to disrupt this defense and seek forced copulations during an extended chase of the ewe. Although this tactic is much less successful for individual rams, those using it can collectively obtain up to $40 \%$ of paternities during a breeding season (Pelletier et al., 2006).

This pattern of sexual coercion being employed by subordinate males and/or opportunistically by all males suggests that the tactic is sub-optimal, probably because the male's prospects of success are usually low in relation to potential costs. Coercion involves a male-female contest which may be costly for the male as well as the female, and may either attract the attention of rival males, thus triggering a malemale contest (Clutton-Brock and Parker, 1995), or render both parties more vulnerable to predation (Evans et al., 2003; Griffiths et al., 2004; Magurran and Nowak, 1991). Copulation, moreover, is only a means to fertilization and without long-term influence over the female, a coercive male's fertilization prospects are more vulnerable to female counter-tactics such as copulation with alternative males (Emlen and Wrege, 1986) or sperm ejection (Pizzari and Birkhead, 2000). Coercion may, nevertheless, be the only option for subordinate males who are attempting to make the most of a bad situation.

Sexual coercion is reported less frequently in birds than in mammals (Caizergues and Lambrechts, 1999; Pradhan and Van Schaik, 2009). As in non-avian taxa, it is nevertheless likely to be adopted opportunistically by males generally or as the only available tactic by subordinates. Among waterfowl (Anatidae) paired, and hence dominant, males are sometimes able to evade the mate-guarding efforts of rival males and force extra-pair copulations (McKinney and Evarts, 1997). While Anatid males possess an intromittent organ, most birds do not (Briskie and Montgomerie, 1997), suggesting that harassment and intimidation are more likely forms of sexual coercion than forced copulation. In free-ranging feral fowl, Gallus gallus domesticus, mating is promiscuous with subordinate males often obtaining copulations by harassment (Lovlie and Pizzari, 2007). Harassment by non-territorial males is also common in ringnecked pheasants, Phasianus colchicus (Mateos, 1998). In many monogamous species, extra-pair males incur no parental care costs and hence have a strong incentive to obtain copulations by any means. The degree to which extra-pair copulations rely on coercion and the degree to which they result in extra-pair paternity, however, are both controversial questions (Westneat and Stewart, 2003).

Australian brush-turkeys, Alectura lathami, provide an interesting case study for avian sexual coercion because coercive male behaviour appears to be ubiquitous in the mating system. Investigation of this behaviour requires placing it in the context of reproduction in the Megapodidae (Birks and Edwards, 2002; Jones and Göth, 2008). Uniquely among birds, megapodes incubate their eggs using environmental sources of heat, usually microbial decomposition in a mound of soil and vegetation (Seymour, 1985). On the basis of this ancestral pattern, variations have evolved. In the brush-turkey system, males construct mounds, control their temperature via ongoing maintenance, defend them against rival males and are polygynous (Jones et al., 1995). Females make frequent visits to mounds, often copulate with the male and periodically dig substantial holes in which they bury their eggs. Females are not subject to mateguarding, do not pair-bond with the male and are polyandrous (Jones et al., 1995). Males evidently use their mound as a means of attracting females interested in the incubation properties of the mound (Jones, 
1992). Having attracted a female, a male typically behaves towards her in a way which suggests coercion, for example by charging at her or pecking her on the back or head (Jones, 1990b).

Male-female aggression is less extreme than that evident when male-male encounters occur near incubation mounds (Jones, 1987), but it is nevertheless substantial, as prolonged interactions can result in the female's death in captive situations where she is unable to escape (Jones et al., 1995). Aggression towards females is interspersed with non-aggressive displays possibly attempting to influence female choice. Where individual females can be confidently identified by the researcher, it is clear that males are attempting to both entice and coerce each female, rather than selectively enticing some females and driving others away. This allows us to exclude male choosiness as an explanation for the coercive behaviour in this species (for more details, see Wells, 2012). The male's aggression probably discourages visits from females unwilling or unable to tolerate it, hence biasing the copulations which occur, but aggression is not usually a form of rejection. Expulsion of a female from the mound is almost always followed by an attempt to entice her back (Wells, 2012).

Sexual coercion is not an unusual animal mating tactic, but brush-turkey males appear to attempt it in unusual circumstances. In the discussion, we outline these circumstances, and propose that the coercion reflects a behavioural syndrome, namely a set of behaviours which is consistent across multiple different contexts, but may have little or no function when considered in one of these contexts in isolation (Sih et al., 2004).

In summary, our objectives can be expressed in the following three questions:

1. Which male behaviours are aggressive, and conversely, which are enticing?

2. If male behaviours can be distinguished as above, do males attempt to obtain copulations coercively?

3. If males are attempting to obtain copulations coercively, to what degree are they successful?

\section{Methods}

Field studies were conducted on a free-living, individually colour-banded brush-turkey population at the town of Pearl Beach in New South Wales, Australia (33.54॰ S, 151.30 E). Birds were captured in baited steel walk-in traps $2.4 \mathrm{~m} \times 1.6 \mathrm{~m}$ and $1 \mathrm{~m}$ high. To minimize the risk of injury, traps were not left unattended. Each bird was removed for banding by entering the trap, securing its legs and placing it into a non-transparent cloth bag.

Observations were recorded remotely by five Sony HDR-SR7 video cameras mounted at active mounds and programmed to run daily for $3.5 \mathrm{~h}$ from first light. Previous observations have shown that nearly all reproductive behaviour occurs during these hours (Jones, 1988). Males in the study population constructed or maintained mounds between July and January. Data for Question 1 are drawn from the 2008-09 breeding season (14 males observed maintaining 17 mounds Nov-Dec 2008, there being three instances of a single male maintaining two mounds). To achieve sufficient statistical power when answering Questions 2 and 3, data from the 2009-10 season were also included (16 males, each male observed maintaining a single mound August-October 2009). With some exceptions due to access difficulties or extreme weather, each mound was observed for seven consecutive days.

When reviewing video of female visits to mounds, behaviour was recorded on an all-occurrences basis to the nearest second. All data collection and video review was conducted by a single observer. The total number of female mound visits was 144 for 2008-09 and 228 for 2009-10 (for details of how female visits were defined, see Supplementary material A). The total number of visiting females individually identifiable 
in the context of a mound was 46 for 2008-09 and 48 for 2009-10 (for details of how females were identified, see Supplementary material B).

Only pre-defined behaviours were recorded (Table 1). Counts were recorded for behaviours of relatively short duration, i.e. events, while the frequency and duration of bouts were recorded for behaviours of relatively long duration, i.e. states (Martin and Bateson, 2007). This distinction was in some cases influenced by context, however (for details see Supplementary material C).

Table 1. Pre-defined behaviours, distinguished according to whether they are performed by males or females and whether they are events or states. The definitions are similar to, but in some cases amplify or simplify those in Jones (1987). The term 'bird-peck' refers to a male behaviour which is here classified as a state, but when answering Questions 2 and 3 is treated as an event, enabling the number of pecks to be counted. The collective term 'female mound activity' refers to female behaviours which either prepare a mound for egg-laying or appear to assess it for future egg-laying.

\begin{tabular}{|c|c|}
\hline Behaviour & Description \\
\hline \multicolumn{2}{|l|}{ Male events } \\
\hline Towards & $\begin{array}{l}\text { Move towards female without running; male's intention remains unclear, either } \\
\text { because the female avoids him or he suspends action }\end{array}$ \\
\hline Failed copulation & $\begin{array}{l}\text { Move towards female to copulate; movement is usually rapid, with neck outstretched; } \\
\text { male may or may not succeed in making significant physical contact with the female; } \\
\text { in the former case, he may almost succeed in copulating }\end{array}$ \\
\hline Rush-and-maintain & $\begin{array}{l}\text { Run, flap wings rapidly or hold in a spread position, and commence rapid digging or } \\
\text { scratching (see 'female mound activity' below for definitions of 'dig' and 'scratch') }\end{array}$ \\
\hline Imminent arrival (female present) & $\begin{array}{l}\text { Female is at the mound while the male owner is absent, and female observes his } \\
\text { approach }\end{array}$ \\
\hline Bird-peck & $\begin{array}{l}\text { Peck with downward stroke to female's tail, body or head, sometimes using bill to } \\
\text { grasp female's neck or head; usually delivered while hovering }\end{array}$ \\
\hline Maintain & Dig, probe or scratch in mound substrate, as defined for 'female mound activity' below \\
\hline Flattened and motionless & $\begin{array}{l}\text { Lower body to mound substrate, and lie with wings out and tail spread (flattened); a } \\
\text { simplified version is to adopt a crouched position, usually in a hole (motionless) }\end{array}$ \\
\hline Ground-peck & Peck repeatedly at the mound substrate but without foraging \\
\hline \multicolumn{2}{|c|}{ Female mound activity (all treated as states) } \\
\hline Dig & $\begin{array}{l}\text { Move substrate with a backward motion of the legs, resulting in commencement or } \\
\text { expansion of a hole }\end{array}$ \\
\hline
\end{tabular}

\subsection{The effect of male behaviour on female mound activity}

By recording both male and female behaviour against the same timescale, it was possible to consider the effect of male behaviour on female behaviour, and hence to test the hypothesis that male behaviour partitioned into aggressive and inviting components. The key observation is that whereas males seemed to focus on exploiting their control over the mound as a way of maximizing the number of copulations 
(Jones, 1990a), females seemed to focus on the qualities of the mound as an incubator, as evidenced by their digging, probing and scratching in the mound, either to prepare a hole for egg-laying or to explore the incubation properties of a mound apparently with a view to future egg-laying (Birks, 1996). The collective term 'female mound activity' will henceforth be used to refer to these female behaviours (Table 1).

Cessation of female mound activity appeared to represent a cost for females because they could no longer assess the quality of the mound or prepare it for egg-laying. Any male behaviour which caused mound activity to cease, therefore, potentially imposed a greater cost and could be classified as aggressive. Conversely, any male behaviour which caused mound activity to commence could be classified as enticing. This more objective approach avoided the anthropomorphic assumption that aggression was any behaviour which 'looked nasty'. Because the female change occurred after the onset of the male behaviour, it was also possible to be more confident about causality. See Supplementary material $D$ for details on how female responses were allocated to male events and states.

When considering the impact on female commencement or cessation of mound activity, commencement was defined narrowly to exclude ambiguous events such as entering a hole without starting to dig. Conversely, cessation was defined narrowly to exclude leaving a hole where there was no prior digging activity, and hence no cessation of digging. Some male behaviours only occurred early during female visits, however, when mound activity was unlikely to have commenced. Female arrivals and departures from the mound were therefore also included as responses, these being respectively precursors to mound activity or confirmation that it had ceased. A mixed effects logistic regression model was used to categorize male behaviours as aggressive, inviting or neither. The data points were instances where any of the pre-specified male behaviours were observed to precede either commencement or cessation of female mound activity (for mathematical details, see Supplementary material E). This analysis ignores dynamic effects, namely the back-and-forth influence of male and female behaviour. We plan an evolutionary game-theoretic study to investigate these questions in more detail.

\subsection{Do males attempt to obtain copulations coercively?}

Four approaches were used to determine whether males were attempting sexual coercion. The first approach was to analyze male behaviour before and after copulation. As assessed by a paired t-test, greater incidence of a behaviour pre- as opposed to post-copulation was taken as evidence that males employed the behaviour as a way of achieving copulation (for a similar approach, see Bro-Jorgensen, 2003). Conversely, reduced incidence of a behaviour pre-copulation might indicate that males avoided the behaviour as a way of achieving copulation. For each category of male behaviour, frequencies or durations were calculated for 1-, 5-, 10- and 20-min intervals both prior to and subsequent to each copulation, and then compared with each other. Preliminary analysis suggested that the 5-min interval should be used in the analysis (for details, see Supplementary material F).

In some circumstances, examination of pre- and post-copulation male behaviour may confuse the analysis. For example, male lemurs, Lemur catta, characteristically self-groom intensively after copulation (Evans and Goy, 1968), but neither the presence of this behaviour post-copulation nor its absence precopulation has any causal influence on the likelihood of copulation. In the brush-turkey case, behaviours which are elevated pre-copulation relative to post-copulation may exhibit this pattern simply because they have been displaced post-copulation by the brush-turkey equivalent of lemur self-grooming. This possibility was tested by using a paired t-test to compare pre- and post-copulation incidences with mean incidences for the entire visit. Only if a behaviour was significantly elevated post-copulation, not only relative to pre-copulation but also relative to the visit mean, was it likely to represent a post-copulation phenomenon. 
The third method used to investigate the impact of male behaviour on copulation was to graph male and female behaviour against visit time in an attempt to identify patterns in the timing of copulations, particularly in relation to male aggression and enticement. To enable both events and states to be shown on the same scale, behaviour was expressed in cumulative percentage terms. Once a pattern was identified, a paired t-test was used to compare the first half of visits to the second half. Subsequently, visits were split at different points to determine which split yielded the strongest contrast.

In addition to these open-ended approaches, specific evidence was sought for each of the three recognized types of sexual coercion. For forced copulation, the female's ability to avoid copulation was assessed by examining video of failed copulations, while the degree of her acquiescence was assessed by examining successful copulations. Unusually among birds, male brush-turkeys possess a phallus, although this is non-intromittent (Brennan et al., 2008), suggesting that forced copulation has a low probability of success. For harassment, the frequencies of attempted and actual copulations were compared. Intimidation was identified as follows: where copulation occurred early in a visit (within the first quarter of visit elapsed time), the preceding visit by the same female at the same mound was examined to determine whether the rate of male aggression was higher than the mean rate for the male.

\subsection{Do males succeed in obtaining copulations coercively?}

A logistic regression model was used to assess aggression and enticement rates as predictors of copulation during female visits where the male was also present. Male identity and whether or not an egg was laid were also included as predictors in the regression (for mathematical details, see Supplementary material G). In addition, aggression and enticement rates were compared for visited males who did vs. did not obtain copulations during the two study seasons (t-test).

\section{Results}

\subsection{The effect of male behaviour on female mound activity}

The log-likelihood for the full mixed effects logistic regression model was -112.36 , and therefore the critical log-likelihood for the nested models at the $5 \%$ significance level was -114.28 . Male behaviours were either significantly more likely to be followed by the cessation of female mound activity and hence could be classified as aggressive, or were significantly more likely to be followed by the commencement of mound activity and hence could be classified as enticing (Table 2). The sole exception to this pattern was the rush-and-maintain behaviour, which was excluded from subsequent analysis. Although male behaviours partitioned into aggression and enticement, this result was obtained by focusing on changes in female behaviour in response to male behaviour. When all effects on female behaviour were considered, including those involving no change, females were more likely to continue rather than alter whatever they were doing prior to the onset of the male behaviour, indicating that males had only a limited ability to influence female behaviour.

\subsection{Do males attempt to obtain copulations coercively?}

Some types of male aggression tended to precede copulation (see behaviours highlighted as 'precopulation aggression', Table 2). Collectively, these behaviours occurred at a significantly higher rate precopulation as opposed to post-copulation for 76 copulations during the 2008-09 season $\left(t_{\text {paired }}=4.432\right.$, $P<0.001)$. Male enticement exhibited a similar pattern $\left(t_{\text {paired }}=5.201, P<0.001\right)$, but both hover

$\left(t_{\text {paired }}=-5.987, P<0.001\right)$ and bird-peck $\left(t_{\text {paired }}=-3.065, P=0.003\right)$ exhibited the opposite pattern (Fig. 1). A single behaviour (the male's imminent arrival with the female already present) exhibited neither pattern and was excluded from subsequent analysis. 
Fig. 1. Mean differences between rates of specified male behaviours for 76 copulations. Pre-copulation behaviours are compared with post-copulation behaviours by subtracting rates for the latter from rates for the former. Error bars represent $95 \%$ confidence intervals.

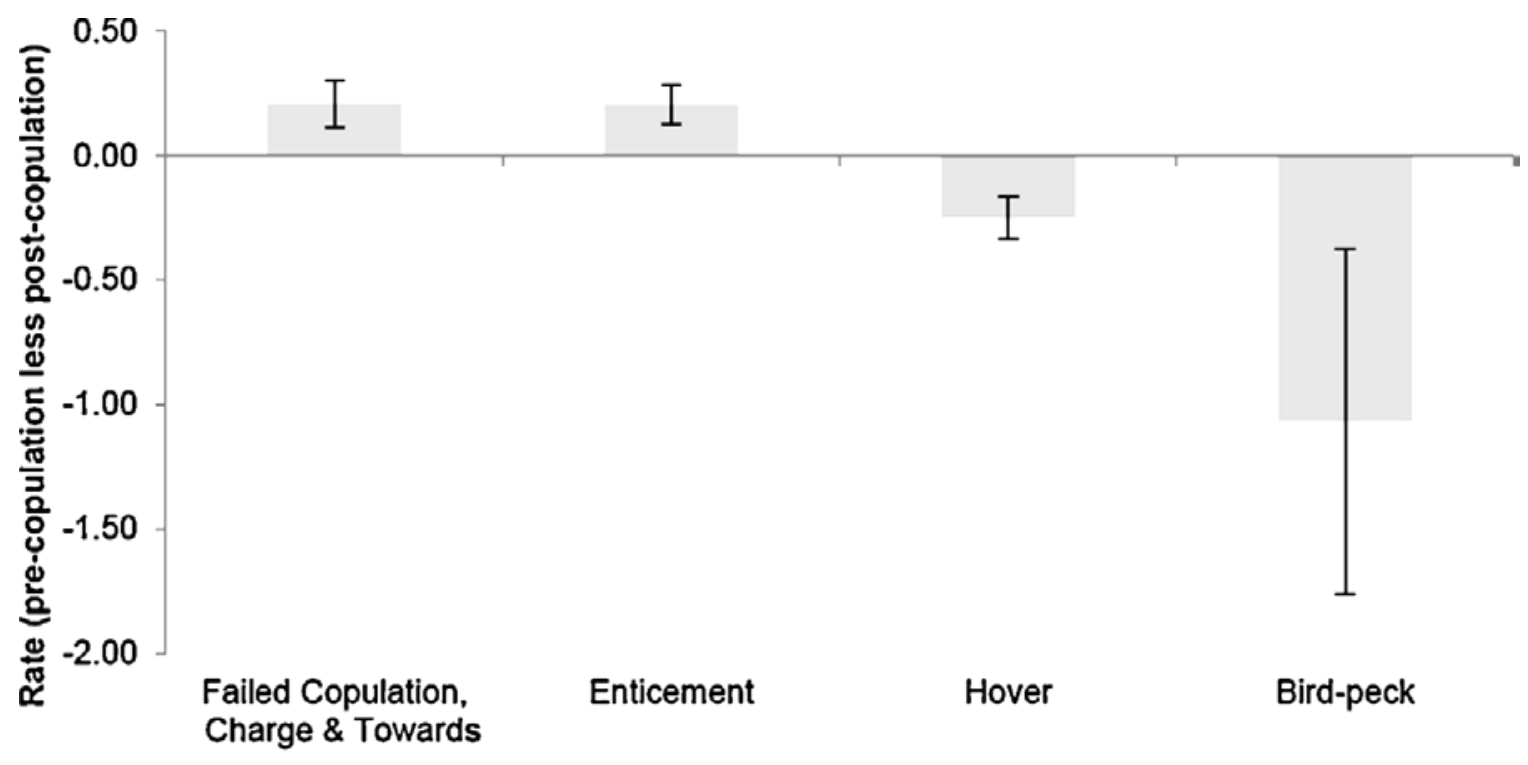

Male behaviour

Fig. 2. Mean differences between rates of specified male behaviours for 82 visits. The first $45 \%$ of visit duration is compared with the remaining $55 \%$ by subtracting rates for the latter from rates for the former. Error bars represent $95 \%$ confidence intervals. Significance should be interpreted with caution, as a single sample was subjected to repeated measures to identify the strongest contrasts overall.

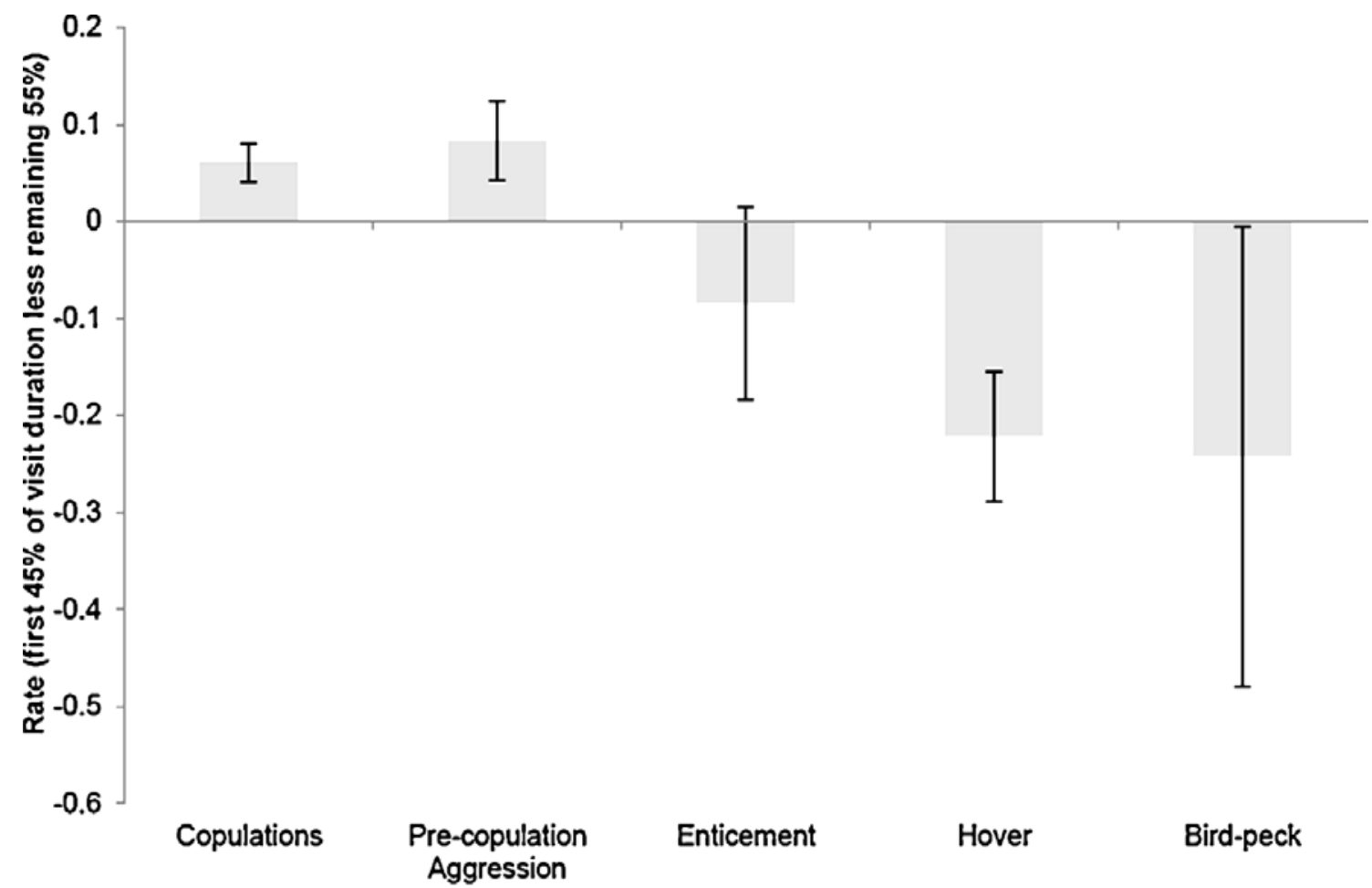

Male behaviour 
Table 2. Results of likelihood ratio tests, categorizing male behaviours as aggressive, enticing or neither. The fixed effects $\mu_{\mathbf{i}}$ represent each male behaviour's tendency (relative to the other male behaviours) to precede commencement as opposed to cessation of female mound activity. Significantly negative fixed effects indicate aggressive male behaviours. Significantly positive fixed effects indicate inviting male behaviours. Insignificant fixed effects are not assumed to be aggressive or inviting.

\begin{tabular}{|lcccc|}
\hline Male behaviour & Estimated effect $\mu_{\mathbf{i}}$ & $\begin{array}{c}\text { Log-likelihood of } \\
\text { nested model }\end{array}$ & $\boldsymbol{p}$ & Conclusion $^{-}$ \\
\hline Towards & -22.09 & -129.27 & $<0.001$ & Aggressive $^{\mathrm{a}}$ \\
Failed copulation & -28.51 & -117.91 & $<0.001$ & Aggressive $^{\mathrm{a}}$ \\
Charge or barge & -22.10 & -132.01 & $<0.001$ & Aggressive $^{\mathrm{a}}$ \\
Rush-and-maintain & -20.98 & -113.05 & 0.2390 & Not significant \\
Imminent arrival (female present) & -24.24 & -117.21 & 0.0018 & Aggressive \\
Hover & -1.97 & -125.04 & $<0.001$ & Aggressive \\
Bird-peck & -2.30 & -116.63 & 0.0035 & Aggressive \\
Maintain & 1.67 & -125.94 & $<0.001$ & Enticing \\
Flattened and motionless & 3.18 & -125.25 & $<0.001$ & Enticing \\
Ground-peck & 1.27 & -122.51 & $<0.001$ & Enticing \\
\hline
\end{tabular}

${ }^{\mathrm{a}}$ Pre-copulation aggression (see Question 2).

When compared with the visit mean, both hover and bird-peck were significantly reduced pre-copulation, but not significantly elevated post-copulation $\left(t_{\text {paired }}=-9.237, P<0.001\right.$ and $t_{\text {paired }}=-5.088, P<0.001$ respectively pre-copulation and $t_{\text {paired }}=-0.781, P=0.437$ and $t_{\text {paired }}=0.809, P=0.421$ respectively post-copulation). This finding of a significant reduction pre-copulation rather than a rise post-copulation suggests that hover and bird-peck were not post-copulation behaviours capable of displacing precopulation aggression and enticement.

With some exceptions, copulations and the male behaviours which tended to precede copulation were more likely to occur earlier during visits, while the male behaviours which tended to follow copulation were more likely to occur later during visits. When visit time was split evenly at the mid-point, rates of copulation $\left(t_{\text {paired }}=6.135, P<0.001\right)$ and enticement $\left(t_{\text {paired }}=2.5, P=0.031\right)$ were significantly elevated in the first 50\%, and there was also a non-significant trend for pre-copulation aggression to be elevated $\left(t_{\text {paired }}=1.827, P=0.098\right)$. Meanwhile, hover was elevated in the second $50 \%\left(t_{\text {paired }}=-2.76, P=0.02\right)$, although bird-peck was not $\left(t_{\text {paired }}=-0.241, P=0.814\right)$. The strongest overall contrasts were found after $45 \%$ of visit time had elapsed (Fig. 2).

Where the male made physical contact with the female during a copulation attempt, but ultimately failed to copulate, this failure could clearly be attributed to avoidance by the female (53 of 54 cases). Where copulation was behaviourally complete, i.e. cloacal contact appeared to be achieved, there was almost always prior movement by the female to expose the cloaca, indicating that she accepted the male (95 of 100 cases, after excluding those in which a clear view was obstructed). The remaining five cases were assumed to be forced copulations. A check for evidence of harassment revealed that males made 277 unsuccessful copulation attempts compared to 122 successful copulations, i.e. a ratio of 2.27:1.

When testing for intimidation, the distinction between egg-laying and non-laying visits was a strong confounding factor. Where copulation occurred early and the preceding visit was not egg-laying $(N=34$ visits with early copulation), rates of male hover and bird-peck aggression during the preceding visit were significantly lower than the mean rate for the male $\left(t_{\text {paired }}=7.116, P<0.001\right.$ and $t_{\text {paired }}=6.848, P<0.001$ respectively). Where copulation occurred early and the preceding visit was egg-laying $(N=$ seven visits 
with early copulation), there was a non-significant trend for the rates of male hover and bird-peck aggression to be higher during the preceding visit than the mean rate for the male $\left(t_{\text {paired }}=-2.007, P=\right.$ 0.092 and $t_{\text {paired }}=-2.354, P=0.057$ respectively). Meanwhile, there was no difference in the rate of precopulation aggression where the preceding visit was not egg-laying $\left(t_{\text {paired }}=-1.166, P=0.252\right)$, but the rate was significantly lower where the preceding visit was egg-laying $\left(t_{\text {paired }}=4.581, P=0.004\right)$. There was, therefore, no evidence that males were employing intimidation as a tactic.

\subsection{Do males succeed in obtaining copulations coercively?}

Male identity was controlled for, but was not a predictor of interest in the logistic regression. Of the other variables, only hover and bird-peck aggression (combined as a single predictor variable) were significant (Table 3). Males with higher rates of pre-copulation aggression were not significantly more likely to copulate $(t=0.474 . P=0.642, N=18$ visited males). Non-significant results were also obtained for hover and bird-peck aggression combined $(t=1.329, P=0$. 203) and enticement $(t=-0.161, P=0.874)$.

\section{Discussion}

With very few exceptions, the behaviour of individual male brush-turkeys during female visits could be classified as either aggressive or enticing (Table 2). Copulation was preceded both by high rates of male enticing behaviour and certain types of aggression (Fig. 1). It appears, therefore, that males were attempting sexual coercion, while simultaneously attempting to attract females to the mound. Our observations suggest that coercion took the form of harassment. Although there were only 2.27 copulation attempts for each successful copulation, the ratio of all instances of pre-copulation aggression to copulations was nearly $6: 1$. If the objective of harassment is to wear down female resistance, and this might be achieved using a wider range of aggressive behaviours than attempted copulation alone, then that wider range of behaviours would seem to qualify as harassment.

There was no evidence, however, that harassment was a successful tactic. Brush-turkey males with a higher rate of pre-copulation aggression were not significantly more likely to copulate, regardless of whether one assessed this likelihood by female visit (Table 3) or by males individually. The co-occurrence of copulations and male pre-copulation aggression during the first $45 \%$ of female visits (Fig. 2) might be counted as evidence for the success of harassment, but it is equally possible that males, observing a female propensity to copulate during this period, intensified their harassment during this period. Apart from a small number of apparently forced copulations, obtained when females were pinned against the side of the hole from which they were attempting to escape, there was no evidence that the use of aggression was successful in obtaining copulations. A strong positive association was nevertheless found between copulation success and two aggressive behaviours, hover and bird-peck (Table 3), which tended to be elevated post-copulation rather than pre-copulation (Fig. 1). A compelling explanation for this paradox is that causality operated in reverse. Post-copulation, males allowed females greater access to the mound. Consequently, females spent more time digging holes in the mound, a relatively stationary activity which enabled these forms of male aggression (Wells, 2012).

Brush-turkey males attempted sexual coercion in unexpected circumstances. Firstly, coercion was attempted despite little evidence for its success. Secondly, it was attempted by males in control of mounds, and hence apparently in a position to exert considerable influence over the female. Thirdly, because mound-ownership is a necessary precondition for membership of the mating pool (Jones, 1990a), coercion was, unusually, employed as a primary mating tactic.

The finding that sexual coercion was employed despite little evidence that the tactic was successful prompted us to search the literature for similar reports. In species where forced copulation is a primary 
tactic, efficacy was hardly in doubt because behaviourally complete copulation could be observed, regardless of whether the species was an invertebrate (Arnqvist, 1989; Parker, 1970; Vahed, 2002) or a vertebrate (Le Boeuf and Mesnick, 1991; Pilastro et al., 1997; Shine and Mason, 2005; Solensky, 2004). The efficacy of amplexus in frogs could also be observed because fertilization is external (Howard, 1980). Where sexual coercion is a secondary tactic, some evidence of success has been found, regardless of whether coercion was practiced as an opportunistic tactic by dominant males (Evans et al., 2003; Lee and Hays, 2004; Low, 2005; McKinney et al., 1983; Westneat, 1987) or as the only available tactic by subordinates (Bro-Jorgensen, 2003; Collias and Collias, 1996; Galdikas, 1985; Pelletier et al., 2006). In some cases, however, efficacy as a tactic for subordinates was difficult to measure, and although therefore unknown, may have been zero or close to zero (Le Boeuf and Mesnick, 1991; Stumpf and Bösch, 2010; Sundaresan et al., 2007; Thiel and Hinojosa, 2003). We found only one case of attempted sexual coercion, together with explicit evidence that the coercion was unlikely to be successful, consistent with the findings reported here. Female white-fronted bee-eaters, Merops bullockoides, are sometimes chased and forcibly mated by extra-pair males, but cloacal contact was recorded in only $3 \%$ of instances where the chase could be fully observed (Emlen and Wrege, 1986).

Table 3. Results of a logistic regression model estimating the likelihood of copulation during 205 female visits where the male was present at the mound during the visit. In addition to male identity and measures of male enticement and aggression, predictors include a dichotomous variable which distinguishes egg-laying visits from non-laying visits.

\begin{tabular}{|c|c|c|c|}
\hline Predictor & Estimated coefficient & Standard error & $p$ \\
\hline$E_{\mathrm{ij}}$ : egg laid & 17.39 & 5033.69 & 0.997 \\
\hline$R_{\mathrm{ij}}^{\mathrm{A}}$ : rate of pre-copulation aggression & -1.09 & 1.08 & 0.314 \\
\hline$R_{\mathrm{ij}}^{\mathrm{U}}:$ rate of hover and bird-peck aggression combined ${ }^{\mathrm{a}}$ & 8.05 & 1.87 & $<0.001$ \\
\hline$R_{\mathrm{ij}}^{\mathrm{E}}$ : rate of enticement & 1.09 & 0.97 & 0.258 \\
\hline
\end{tabular}

${ }^{a}$ Hover and bird-peck were combined into a single variable to minimize the number of predictors in the model (these behaviours have similar characteristics - see Section 3).

Forced copulations by brush-turkey males appeared to have similarly low prospects of success, representing only $1.25 \%$ of all copulation attempts. Just as forced copulation by male bee-eaters has been interpreted as a low yield/low cost tactic pursued opportunistically by monogamously mated males who must be present in the colony in order to feed their mates (Emlen and Wrege, 1986), so one might argue that brush-turkey males must be present at their mounds, and hence can take advantage of the reliable presence of females. The difficulty with this argument, however, is that a low success rate does not appear to be matched by a similarly low cost. Males expended considerable effort on sexual harassment, a form of coercion which clearly made females more wary, and hence apparently less rather than more likely to become victims of forced copulation. Harassment works by wearing down resistance, so that females eventually agree to copulate. This, rather than forced copulation, was the tactic which appeared to be unsuccessful.

Resource defence is a frequent tactic employed by males seeking mating opportunities, but vertebrates do not often combine it with coercion. Our search of the literature found only three instances: Topi antelope, Damaliscus lunatus (Bro-Jorgensen, 2003), Grevy's zebra, Equus grevyi (Sundaresan et al., 2007) and elephant seals, Mirounga angustirostris (Le Boeuf and Mesnick, 1991; Mesnick and Le Boeuf, 1991). In the first two cases, a combination of resource defence and coercion was a secondary tactic employed by subordinate males who either could not secure a place at a breeding lek (topi) or were 
unable to establish longer-term relationships with females (zebra). Only elephant seals conformed to the pattern of resource defence in combination with coercion as a primary tactic, as reported here.

Sexual coercion in combination with mound-ownership was clearly a primary tactic in brush-turkeys. Not only was coercion attempted by dominant mound-owning males, there was no evidence that it was employed by subordinate non-owners. Indeed, there is little evidence for any copulation away from mounds. (but see Dow, 1988 for an exception). Moreover, non-owning males are rejected by females when they masquerade as mound-owners while the actual owner is absent (Jones, 1990a), suggesting that females are also likely to reject them when encounters occur away from mounds. It therefore seems that mound ownership is so essential for male mating success that non-owning males either do not attempt coercion, or the success rate is negligible. Our search of the literature found only five vertebrate examples of sexual coercion as a primary tactic, as reported here: garter snakes (Shine and Mason, 2005), elephant seals (Le Boeuf and Mesnick, 1991), Gambusia (Pilastro et al., 1997), feral fowl (Lovlie and Pizzari, 2007) and amplexus in frogs (Howard, 1980).

It is tempting to suppose that, given the possibility that females were visiting and copulating with rival males, brush-turkey males were attempting to ensure paternity by enforcing a high copulation rate. Similar paternity concerns must, however, motivate males in other species employing resource defence polygyny, and as we have seen, sexual coercion is nevertheless uncommon in such systems. Moreover, there is strong evidence that brush-turkey females focus their mound visits on a primary male, and are unlikely to copulate with rival males during the visiting period (Wells, 2012). Another possible explanation is that females prefer more aggressive males, but this hypothesis has not received support (Wells, 2012).

A plausible explanation for the behaviour of male mound-owners, we suggest, is the prevalence of agonistic relations in brush-turkey social life generally (Jones, 1987), which provides a bias towards aggression as the default solution to social conflict, including conflict over mating. As for all megapodes, brush-turkey chicks lead an independent existence from the time of hatching and generally do not aggregate on an ongoing basis until they have become juveniles (Göth and Jones, 2003). In contrast to most other megapodes, which are socially monogamous (Jones et al., 1995), no subsequent experience provides a countervailing influence for brush-turkeys, as no pair-bond ever develops. To an unusual degree, therefore, brush-turkey social life is marked by conflict rather than cooperation. In short, we propose a behavioural syndrome (Sih et al., 2004) in which aggression becomes the default solution to social conflict. One advantage of this hypothesis is that it is not specific to sexual coercion, and is therefore broad enough to account for other ways in which aggression is central to brush-turkey social life. In particular, the aggressive behaviour which dominates egg-laying visits appears to be independent of sexual coercion (Wells, 2012).

\section{Acknowledgements}

We thank Geoff Ross of NSW National Parks and Wildlife Service for banding assistance, supply of capture and banding equipment and access to a banded population. Colin McKenzie built the camera systems and Greg Yates supplied the electronics. Ann Göth provided early advice which productively steered us in the direction of field rather than captive research, and many Pearl Beach residents kindly gave access to their properties. Eduardo Gallo-Cajiao, Rowan McGinley, Heather Baldwin, Yagiz Aksoy, Hilal Varinli, Margaret Morgan, Matt Saunders and Upma Dutt provided field assistance. This research was supported by a Macquarie University Research Excellence Scholarship. The funding body had no involvement in designing, carrying out or reporting the study. Permits were granted by Macquarie University Ethics Committee, the Australian Bird and Bat Banding Scheme and NSW National Parks and Wildlife Service. 


\section{References}

Arnqvist, G., 1989. Sexual selection in a water strider: the function, mechanism of selection and heritability of a male grasping apparatus. Oikos 56, 344-350.

Birks, S., (doctoral dissertation) 1996. Reproductive behavior and paternity in the Australian brush-turkey Alectura lathami. Cornell University, Ithaca, NY, USA.

Birks, S., Edwards, S., 2002. A phylogeny of the megapodes (Aves: Megapodiidae) based on nuclear and mitochondrial DNA sequences. Mol. Phylogenet. Evol. 23, 408-421.

Brennan, P., Birkhead, T., Zyskowski, K., van der Waag, J., Prum, R., 2008. Independent evolutionary reductions of the phallus in basal birds. J. Avian Biol. 39, 487-492.

Briskie, J., Montgomerie, R., 1997. Sexual selection and the intromittent organ of birds. J. Avian Biol. 28, 73-86.

Bro-Jorgensen, J., 2003. No peace for estrous topi cows on leks. Behav. Ecol. 14, 521-525.

Caizergues, A., Lambrechts, M., 1999. Male macho mammals exploiting females versus male Don Juan birds exploited by females: the opposite-sex exploitation (OSEX) theory. Ecol. Lett. 2 (4), 204-206.

Clutton-Brock, T., Parker, G., 1995. Sexual coercion in animal societies. Anim. Behav. 49, 1345-1365.

Collias, N., Collias, E., 1996. Social organization of a red junglefowl, Gallus gallus, population related to evolution theory. Anim. Behav. 51, 1337-1354.

Dow, D., 1988. Sexual interactions by Australian brush-turkeys away from the incubation mound. EMU 88, 49-50.

Emlen, S., Wrege, P., 1986. Forced copulations and Intra-specific parasitism: two costs of social living in the white-fronted bee-eater. Ethology 71, 2-29.

Evans, C., Goy, R., 1968. Social behaviour and reproductive cycles in captive ring-tailed lemurs (Lemur catta). J. Zool. 156, 181-197.

Evans, J., Pilastro, A., Ramnarine, I., 2003. Sperm transfer through forced matings and its evolutionary implications in natural guppy (Poecilia reticulata) populations. Biol. J. Linn. Soc. 78, 605-612.

Galdikas, B., 1985. Subadult male orangutan sociality and reproductive behavior at Tanjung Puting. Am. J. Primatol. 8, 87-99.

Göth, A., Jones, D., 2003. Ontogeny of social behavior in the megapode Australian brush-turkey (Alectura lathami). J. Comp. Psychol. 117 (1), 36-43.

Griffiths, S., Brockmark, S., Hojesjo, J., Johnsson, J., 2004. Coping with divided attention: the advantage of familiarity. Proc. R. Soc. Lond. B - Biol. Sci. 271 (1540), 695-699.

Howard, R., 1980. Mating behaviour and mating success in woodfrogs, Rana sylvatica. Anim. Behav. 28, 705-716.

Jones, D., (PhD) 1987. Behavioural ecology of reproduction in the Australian Brush-Turkey Alectura lathami. Griffith, Nathan. 
Jones, D., 1988. Construction and maintenance of the incubation mounds of the Australian brush-turkey Alectura lathami. EMU 88, 210-218.

Jones, D., 1990a. Male mating tactics in a promiscuous megapode: patterns of incubation mound ownershi. Behav. Ecol. 1, 107-115.

Jones, D., 1990b. Social organization and sexual interactions in Australian Brush-turkeys (Alectura lathami): implications of promiscuity in a mound-building megapode. Ethology 84, 89-104.

Jones, D., 1992. An evolutionary approach to megapode mating systems. In: Paper Presented at the Proceedings of the First International Megapode Symposium, Christchurch.

Jones, D., Dekker, R., Roselaar, C., 1995. The Megapodes. Oxford University Press, Oxford.

Jones, D., Göth, A., 2008. Mound-Builders. CSIRO Publishing, Melbourne.

Le Boeuf, B., Mesnick, S., 1991. Sexual behavior of male northern elephant seals: I. Lethal injuries to adult females. Behaviour 116, 143-162.

Lee, P., Hays, G., 2004. Polyandry in a marine turtle: females make the best of a bad job. Proc. Natl. Acad. Sci. 101, 6530-6535.

Lovlie, H., Pizzari, T., 2007. Sex in the morning or in the evening? Females adjust daily mating patterns to the intensity of sexual harassment. Am. Nat. 170 (1), E1-E13.

Low, M., 2005. Female resistance and male force: context and patterns of copulation in the New Zealand stitchbird Notiomystis cincta. J. Avian Biol. 36, 436-448.

Magurran, A., Nowak, M., 1991. Another battle of the sexes: the consequences of sexual asymmetry in mating costs and predation risk in the guppy, Poecilia reticulata. Proc. R. Soc. Lond. B - Biol. Sci. 246, 31-38.

Martin, P., Bateson, P., 2007. Measuring Behaviour: An introductory Guide. Cambridge University Press, Cambridge.

Mateos, C., 1998. Sexual selection in the ring-necked pheasant: a review. Ethology Ecology and Evolution 10, 313-332.

McKinney, F., Derrickson, S., Mineau, P., 1983. Forced copulation in waterfowl. Behaviour 86, 250-294.

McKinney, F., Evarts, S., 1997. Sexual coercion in waterfowl and other birds. Ornithol. Monogr. 49, 163195.

Mesnick, S., Le Boeuf, B., 1991. Sexual behavior of male northern elephant seals: II. female response to potentially injurious encounters. Behaviour 117, 262-280.

Parker, G., 1970. The reproductive behaviour and the nature of sexual selection in Scatophaga stercoraria L. (Diptera: Scatophagidae) I. Diurnal and seasonal changes in population density around the site of mating and oviposition. J. Anim. Ecol. 39, 185-204.

Pelletier, F., Hogg, J., Festa-Bianchet, M., 2006. Male mating effort in a polygynous ungulate. Behav. Ecol. Sociobiol. 60, 645-654. 
Pilastro, A., Giacomello, E., Bisazza, A., 1997. Sexual selection for small size in male mosquitofish (Gambusia holbrooki). Proc. R. Soc. Lond. B - Biol. Sci. 264, 1125-1129.

Pizzari, T., Birkhead, T., 2000. Female feral fowl eject sperm of subdominant males. Nature 405, 787789.

Pradhan, G., Van Schaik, C., 2009. Why do females find ornaments attractive? The coercion-avoidance hypothesis. Biol. J. Linn. Soc. 96 (2), 372-382.

Seymour, R., 1985. Physiology of megapode eggs and incubation mounds. Paper presented at the Acta XZIII Congressus Internationalis Ornithologici.

Shine, R., Mason, R., 2005. Does large body size in males evolve to facilitate forcible insemination? A study on garter snakes. Evolution 59, 2426-2432.

Sih, A., Bell, A., Johnson, J., 2004. Behavioral syndromes: an ecological and evolutionary overview. Trends Ecol. Evol. 19 (7), 372-378.

Smuts, B., Smuts, R., 1993. Male aggression and sexual coercion of females in nonhuman primates and other mammals: evidence and theoretical implications. Adv. Study Behav. 22, 1-63.

Solensky, M., 2004. The effect of behavior and ecology on male mating success in overwintering monarch butterflies (Danaus plexippus). J. Insect Behav. 17, 723-743.

Stumpf, R., Bösch, C., 2010. Male aggression and sexual coercion in wild West African chimpanzees, Pan troglodytes verus. Anim. Behav. 79, 333-342.

Sundaresan, S., Fischhoff, I., Rubenstein, D., 2007. Male harassment influences female movements and associations in Grevy's zebra (Equus grevyi). Behav. Ecol. 18, 860-865.

Thiel, M., Hinojosa, I., 2003. Mating behavior of female rock shrimp Rhynchocinetes typus (Decapoda: Caridea)-indication for convenience polyandry and cryptic female choice. Behav. Ecol. Sociobiol. 55, 113-121.

Trivers, R., 1972. Parental investment and sexual selection. In: Cambell, B. (Ed.), Sexual Selection and the Descent of Man 1871-1971. Aldine-Atherton, Chicago, pp. 136-179.

Vahed, K., 2002. Coercive copulation in the alpine bushcricket Anonconotus alpines Yersin (Tettigoniidae: Tettigoniinae: Platycleidini). Ethology 108, 1065-1075.

Wells, D., (doctoral dissertation) 2012. Mating behaviour of the Australian brush-turkey. Macquarie University, Sydney, Australia.

Westneat, D., 1987. Extra-pair copulations in a predominantly monogamous bird: observations of behaviour. Anim. Behav. 35, 865-876.

Westneat, D., Stewart, I., 2003. Extra-pair paternity in birds: causes, correlates, and conflict. Ann. Rev. Ecol. Evol. Syst. 34, 365-396. 